\title{
IDEALS GENERATED BY THREE ELEMENTS
}

\author{
PETER KOHN
}

Abstract. Let $R$ be a commutative Noetherian ring. Any finitely generated module's projective dimension is realized as the projective dimension of some cyclic module with three relations. This result was previously known for local rings.

1. Introduction. We say an ideal is triply generated when three (or fewer) elements generate it. We will use $R$-sequences in global rings and the notation for grade, $G(A)$, according to, for instance, Kaplansky [4, pp. $84 \mathrm{ff}$.; note exercises $11,12,23$ of $\S 3-1]$. For projective dimension we use $d(A)$, subscripted when necessary to identify the ring.

A paper by Burch [2] shows that if $0 \leqq s \leqq G(R)$, where $R$ is local, then for some triply generated ideal $I, d_{R}(R / I)=s$. Furthermore, if the local ring is not regular, then some triply generated ideal has infinite dimension. We discovered how to apply Burch's proof of the infinite case to the finite case, unifying the methods. Better yet, the unified method is applied to global rings in the finite case. (The local infinite result gives the global infinite result.) The preceding is what we present in this paper. Our basic lemmas are in the next section, and the theorems are proved in the section after.

THEOREM A. Let $A$ be a finitely generated module of the commutative Noetherian ring $R, d(A)=h$ (possibly infinite). Then for some triply generated ideal $I, d(R / I)=h$.

2. Reducing generating sets. Given certain types of sets of elements from the (always commutative) ring $R$, we produce smaller sets such that all generated ideals in sight have the same dimension.

Proposition. Let $X, a, b, c, d$ be a sequence of $n$ elements from the ring $R$ (so $X$ is a subsequence and $n \geqq 4$ ) such that every proper subsequence constitutes an $R$-sequence in any order and $d((X, a, b, c, d))>n-2$. Then

(i) $d((X, a b, c d, a c+b d))=d((X, a, b, c, d))$,

(ii) $X, a b, c d, a c+b d$ is a sequence of $n-1$ elements of $R$, and

(iii) $X, a b, c d$ is an $R$-sequence in any order.

Received by the editors November 24, 1971 .

AMS 1970 subject classifications. Primary 13D05, 13E05; Secondary 13C15.

Key words and phrases. Noetherian ring, homological dimension, triply generated ideals, associated primes, $R$-sequence.

c. American Mathematical Society 1972 
Proof. We need the assurance that everything occurs in some proper ideal. For this, note that $d(X, a, b, c, d)>0$. The truth of (ii) is clear. We prove (iii). Let ( $)^{\prime}$ be a permutation so that $(X, a b, c d)^{\prime}$ is in the order in which we are to show it an $R$-sequence. By hypothesis $(X, a, c)^{\prime}$ and $(X, b, c)^{\prime}$ are $R$-sequences. Therefore $(X, a b, c)^{\prime}$ and $(X, a b, d)^{\prime}$ are $R$ sequences, and so $(X, a b, c d)$ is an $R$-sequence, establishing (iii). As a consequence, we record, $d((X, a b, c d))=n-3$. Using \#( ) for number of, this is because \#(X,ab,cd)=n-2 [4, Exercise 1, p. 127].

In order to prove (i) we will calculate the dimension of $(X, a b, c d)$ : $a c+b d$. To do this, we show it equal to $(X, a, c) \cap(X, b, d)$. So, let $r \in(X, a b, c d): a c+b d$. From $r a c+r b d \in(X, a b, c d)$, transposing and selecting, $r a c \in(X, b, d)$. Arguments by now familiar show $X, b, d, a c$ is an $R$-sequence, so $r \in(X, b, d)$. Similarly $r \in(X, a, c)$. The other inclusion remains. If $r$ is in the intersection, then by virtue of the term $(X, b, d)$, $r a c \in(X, a b, c d)$. Likewise $r b d \in(X, a b, c d)$. That is, $r(a c+b d) \in(X, a b, c d)$, so our ideals are equal. Set

$$
K=(X, a, c) \cap(X, b, d)=(X, a b, c d): a c+b d .
$$

Let $m=d((X, a, b, c, d))$, possibly infinite. Consider the exact sequence

$$
0 \rightarrow K \rightarrow(X, a, c) \oplus(X, b, d) \rightarrow(X, a, c)+(X, b, d) \rightarrow 0,
$$

where the injection is $k \mapsto(k,-k)$ and the surjection is $(u, v) \mapsto u+v$. We work from the right. The image on the right is just $(X, a, b, c, d)$ with dimension $m$. In the middle each component is an ideal generated by an $R$-sequence so that each has dimension $n-3$, and so the middle has dimension $n-3$. From hypothesis, $m>n-2>n-3$, so $d(K)=m-1$ [1, 1.6.8 p. 39] or [5, Theorem 2, p. 169].

Now consider the exact sequence

$$
0 \rightarrow(X, a b, c d) \rightarrow(X, a b, c d, a c+b d) \rightarrow R / K \rightarrow 0,
$$

with standard maps. From our latest calculation, $d(R / K)=m$. Previously we recorded $d(X, a b, c d)=n-3$. By hypothesis $m>(n-3)+1$. So, $d(X, a b, c d, a c+b d)=m$, which proves (i).

The last element of the sequence $X, a b, c d, a c+b d$ given by the proposition now needs to be changed if the sequence is to satisfy the proposition's hypotheses, permitting an iteration. This change can be made on the condition, which we henceforth assume, that the ring be Noetherian.

LEMMA 1. Suppose the set $Y$ is an $R$-sequence in any order on the ring $R$, $x \in R$, and $(Y, x) \neq R$. Then for some $y \in R,(Y, y)=(Y, x)$ and every proper subset of $Y$, is an $R$-sequence in any order. 
Proof. First we choose $y$. Let $W$ be any proper subset of $Y$ (allowing $W=\varnothing)$, so some $y_{0} \in Y-W$. Since $y_{0}$ at will follows the elements of $W$ in an $R$-sequence from $Y, y_{0} \notin \mathscr{Z}(R /(W))$ and hence $(Y, x) \nsubseteq \mathscr{Z}(R /(W))$. $(\mathscr{Z}(A)$ is the set of zero-divisors on a module $A$.) Therefore, if $P$ is any maximal prime of zero-divisors on any such $R /(W),(Y, x) \nsubseteq P$. Since the number of all such prime ideals is finite, $(Y, x)$ is not in their union. Consequently, for some $i \in(Y)$, the same is true of $x+i$, that is $x+i \in$ $\mathscr{Z}(R /(W))[4$, Theorem 124 , p. 90]. Set $y=x+i$. Immediately, $(Y, x)=$ $(Y, y)$.

Consider a sequence from a proper subset of $Y, y$. If $y$ is not in it, the sequence is from a subset of $Y$ and so is an $R$-sequence. If $y$ is in the sequence, then the sequence omitting $y$ is from a proper subset of $Y$, so the sequence with $y$ pushed to the end is an $R$-sequence. We can restore $y$ 's position by interchanges of neighbors: If $U, u, y, V$ is known to be an $R$-sequence, then so is $U, y, u, V[4$, Theorem 118, p. 86], thanks to choice of $y$.

This completes the proof of our first lemma. If one does similar things, omitting the word "proper", one obtains the proof of our next lemma.

Lemma 2. Given an $R$-sequence in one order, the ideal it generates can be generated by an $R$-sequence in any order of the same length [4, Exercise 23, p. 104].

Suppose given an $R$-sequence in one order and another element, together generating an ideal of "large enough" dimension: By Lemma 2 on the $R$-sequence followed by Lemma 1, we can satisfy the proposition unless we are handling "too few" elements. The proposition yields a set of elements fewer in number by one, so the dimension is still large enough. We no longer need Lemma 2 after the first application. We explain "large" and "few".

COROllary. Let $N \subseteq R$ be a finite set which, with one element deleted, is an $R$-sequence in one order. If $d((N))>\#(N)-2$, then there exists a triply generated ideal $I, d(I)=d((N))$.

3. Proof of theorem and remarks. We prove the theorem in cases according to $h$, the desired dimension of $R / I$. The case $h=0$ is trivial.

Let $0<h<\infty$. It is well known that then there is an $R$-sequence on $R$ of length $h$. (For instance, see [1, III.6.6, p. 124] and [4, Theorems 173 and 135].) Let $N$ be the $R$-sequence. By the corollary, some triply generated ideal $I$ has the same dimension as $(N)$. That is, $d(R / I)=h$.

We are left with the case of infinite dimension, $h$. The existence of such a module means that $R$ is nonregular globally or, more to the point, for some maximal ideal $M$ the local ring $R_{M}$ is nonregular and $d_{R_{M}}\left(M_{M}\right)=\infty$. 
(Consult [1, loc. cit.] and [5, p. 184].) Choose a maximal $R$-sequence $X$ in $M_{M}$ on $R_{M}$. Then for some $u \in M_{M}-(X), M_{M}=(X): u$. By examining $(X, u) /(X)$ we find $d_{R_{M}}((X, u))=\infty$. With $N=X, u$, our corollary is satisfied on $R_{M}$. This is the case previously known [2]. The triply generated ideal of $R_{M}$ so obtained is easily restricted to a triply generated, infinite dimensional ideal of $R$. Q.E.D.

The theorem has various statements, actually. There is the global version of Burch's statement in terms of grade and regularity. We also record the B version.

TheOREM B. Let $J$ be an ideal in a Noetherian ring. For some triply generated ideal $I, d(I)=d(J)$.

Since one must take the module $R / J$ anyway to prove the finite case of the B version (get a long enough $R$-sequence), we did the A version.

In whatever version, the conclusion cannot be strengthened to yield a doubly generated ideal. Rather, such a theorem is as false as it could be: It is known that if a doubly generated ideal $I$ in a Noetherian ring $R$ has finite dimension then $d(R / I) \leqq 2$. (See MacRae [6]. If $R$ is a UFD, this bound is easy.)

We record that Gulliksen's main theorem in [3] easily follows from Burch's.

This paper is from my thesis, written under Irving Kaplansky.

\section{REFERENCES}

1. H. Bass, Algebraic K-theory, Math. Lecture Notes, Benjamin, New York, 1968. MR 40 \#2736.

2. L. Burch, A note on the homology of ideals generated by three elements in local rings, Proc. Cambridge Philos. Soc. 64 (1968), 949-952. MR 37 \#6278.

3. T. Gulliksen, Tout idéal premier d'un anneau noethérien est associé à un idéal engendré par trois éléments, C. R. Acad. Sci. Paris Sér. A-B 271 (1970), A1206-A1207.

4. I. Kaplansky, Commutative rings, Allyn and Bacon, Boston, Mass., 1970. MR 40 \#7234.

5. - - Fields and rings, Univ. of Chicago Press, Chicago, Ill., 1969. MR 42 \#4345.

6. R. E. MacRae, On an application of the Fitting invariants, J. Algebra 2 (1965), 153-169. MR 31 \#2296.

Department of Mathematics, University of Chicago, Chicago, Illinois 60637

Current address: Department of Mathematics, Indiana University, Bloomington, Indiana 47401 